\title{
Quantum thermal transport from classical molecular dynamics
}

\author{
Jian-Sheng Wang* \\ Center for Computational Science and Engineering, and Department of Physics, \\ National University of Singapore, Singapore 117542, Republic of Singapore
}

(Dated: 22 June 2007)

\begin{abstract}
Using a generalized Langevin equation of motion, quantum ballistic thermal transport is obtained from classical molecular dynamics. This is possible because the heat baths are represented by random noises obeying quantum Bose-Einstein statistics. The numerical method gives asymptotically exact results in both the low-temperature ballistic transport regime and high-temperature strongly nonlinear classical regime. The method can be thought of as a semi-classical approximation to the quantum transport problem. A one-dimensional quartic on-site model is used to demonstrate the crossover from ballistic to diffusive thermal transport.

PACS numbers: 05.60.Gg, 44.10.+i, 63.22.+m, 65.80.+n
\end{abstract}

Many approaches have been used to study lattice heat transport in bulk materials and nanostructures. For bulk materials, the standard method is that of Peierls based on Boltzmann equation for phonons [1, 2]. For quasione-dimensional systems and nanojunctions, a variety of techniques has been used, such as molecular dynamics (MD) 3, 4], mode-coupling theory [5], nonequilibrium Green's function (NEGF) method 6, 7, 8, 9, 10, 11], Schrödinger equation method [12], quantum Langevin dynamics [13, 14], rigorous Boltzmann equations [15], etc. One of the outstanding problems in heat transport is to reconcile the ballistic nature at low temperatures and diffusive transport at high temperatures. As far as we know, the methods mentioned above work only in either ballistic regime, or diffusive regime, but none correctly in both.

Molecular dynamics has the potential to be such a universal method for heat transport. However, since MD is based on classical Newtonian mechanics, the quantum effect is completely absent. Thus we can not expect that it is still correct at low temperatures. In fact, due to very high Debye temperatures for carbon based materials, even $300 \mathrm{~K}$ is considered a low temperature. The kinetic theory of heat transport for phonons gives a formula for the thermal conductivity as $\kappa=\frac{1}{3} c v l$, where $c$ is heat capacity, $v$ is sound velocity, and $l$ is mean free path. The reduction of thermal conductivity at low temperatures is mainly due to much reduced quantum heat capacity $c$, but a classical MD can only produce a constant heat capacity.

Can we simulate a quantum system within MD? At first sight, this seems impossible, since classical dynamics can only produce classical results. In this paper, we show that the heat transport problem in junction systems can be studied with a classical generalized Langevin dynamics using a quantum heat bath derived from BoseEinstein statistics. Instead of the generic Nosé-Hoover heat bath, it is essential to use the generalized Langevin dynamics with memory kernel and colored noises to take care correctly the effect of the baths. The heat baths are modeled as infinite numbers of coupled harmonic oscillators. A remarkable feature of the proposed dynamics is that it reproduces the quantum ballistic results at low temperature when nonlinearity can be neglected, as well as gives a correct high-temperature, strongly nonlinear result. This appears to be the only method that is numerically exact in both limits. Although the classical and quantum generalized Langevin equations are wellknown, it is somewhat surprising that they are seldom used in molecular dynamics. In fact, they have much better properties with respect to heat baths; we advocate their use for thermal transport problems. Our method is inspired by the NEGF approach [10] to heat transport and also the quantum Langevin approach [14] to the same problem.

In the rest of the paper, we first introduce the model and give the equations involved. We then compare the MD results with Landauer formula and with the nonlinear NEGF results. We treat a one-dimensional (1D) quartic nonlinear onsite model, in which we have seen ballistic transport at temperatures below $200 \mathrm{~K}$, and diffusive transport about $1000 \mathrm{~K}$ for lattice sizes up to 4096 .

The general setup of our system consists of a central junction region connected to two semi-infinite harmonic lattices which serve as leads. The Hamiltonian of the system is

$$
\mathcal{H}=\sum_{\alpha=L, C, R} H_{\alpha}+\left(u^{L}\right)^{T} V^{L C} u^{C}+\left(u^{C}\right)^{T} V^{C R} u^{R}+H_{n},
$$

where $H_{\alpha}=\frac{1}{2}\left(\dot{u}^{\alpha}\right)^{T} \dot{u}^{\alpha}+\frac{1}{2}\left(u^{\alpha}\right)^{T} K^{\alpha} u^{\alpha}, u^{\alpha}$ is a column vector consisting of all the displacement variables in region $\alpha(=\mathrm{L}, \mathrm{C}, \mathrm{R})$, and $\dot{u}^{\alpha}$ is the corresponding conjugate momentum. The superscript $T$ stands for matrix transpose. We have chosen a renormalized displacement $u_{j}=\sqrt{m_{j}} x_{j}$ where $m_{j}$ is the mass associated with $j$-th degree of freedom, $x_{j}$ is the actual displacement having the dimension of length. $K^{\alpha}$ is the spring constant matrix and $V^{L C}=\left(V^{C L}\right)^{T}$ is the coupling matrix of the left lead to the central region; similarly for $V^{C R}$. The 
equations of motions are of the form

$$
\begin{aligned}
\ddot{u}^{C} & =-K^{C} u^{C}+F_{n}\left(u^{C}\right)-V^{C L} u^{L}-V^{C R} u^{R}, \\
\ddot{u}^{L} & =-K^{L} u^{L}-V^{L C} u^{C} \\
\ddot{u}^{R} & =-K^{R} u^{R}-V^{R C} u^{C}
\end{aligned}
$$

The heat-bath degrees of freedom $u^{L}$ and $u^{R}$ can be eliminated by solving them in terms of the central variables and initial conditions, given, e.g., for the left lead:

$$
\begin{aligned}
u^{L}(t)= & \int_{t_{0}}^{t} g\left(t, t^{\prime}\right) V^{L C} u^{C}\left(t^{\prime}\right) d t^{\prime} \\
& +\frac{\partial g\left(t, t_{0}\right)}{\partial t_{0}} u^{L}\left(t_{0}\right)-g\left(t, t_{0}\right) \dot{u}^{L}\left(t_{0}\right),
\end{aligned}
$$

where $g\left(t, t^{\prime}\right)$ is the time-domain retarded surface Green's function of the left lead obtained by the solution of

$$
\frac{\partial^{2} g\left(t, t^{\prime}\right)}{\partial t^{\prime 2}}+g\left(t, t^{\prime}\right) K^{L}=-\delta\left(t-t^{\prime}\right) I
$$

with the condition $g\left(t, t^{\prime}\right)=0$ if $t-t^{\prime} \leq 0$.

Substituting the formal solutions of the leads into the central region, we obtain the following generalized Langevin equation [14, 16, 17] for the central part of the degrees of freedom:

$$
\ddot{u}^{C}=-K^{C} u^{C}+F_{n}\left(u^{C}\right)-\int_{t_{0}}^{t} \Sigma\left(t, t^{\prime}\right) u^{C}\left(t^{\prime}\right) d t^{\prime}+\xi_{L}+\xi_{R},
$$

where $F_{n}$ is the nonlinear force, $\Sigma$ is the retarded selfenergy of the leads, $\Sigma=\Sigma_{L}+\Sigma_{R}$, as used in the NEGF calculation, but in the time domain; $\Sigma_{L}=V^{C L} g V^{L C}$. A similar equation holds for the right lead $\Sigma_{R}$ using the right lead surface Green's functions. Contribution from the left lead due to the initial conditions is

$$
\xi_{L}(t)=V^{C L}\left(g\left(t, t_{0}\right) \dot{u}^{L}\left(t_{0}\right)-\frac{\partial g\left(t, t_{0}\right)}{\partial t_{0}} u^{L}\left(t_{0}\right)\right) .
$$

The expression for the right lead $\xi_{R}$ is analogous. The initial time $t_{0}$ will be set to $-\infty$. Using the concept of adiabatic switch-on, at time $-\infty$, the three subsystems, left lead, central region, and right lead, are decoupled and the leads are in respective thermal equilibrium. We turn Eq. (7) into a stochastic differential equation by requiring that $u^{L}\left(t_{0}\right)$ and $\dot{u}^{L}\left(t_{0}\right)$ are random variables.

So far we have treated the system as a classical system. However, at this point, we'll make a departure and treat the leads quantum-mechanically. Since the lead system is linear, the classical equation of motion and quantum Heisenberg equation of motion are identical. At time $t_{0} \rightarrow-\infty$, the leads are isolated. We assume that the leads obey a quantum Bose-Einstein statistics. This induces a random variable $\xi_{L}(t)$ having zero mean, $\left\langle\xi_{L}(t)\right\rangle=0$, and the following correlation matrix

$$
\left\langle\xi_{L}(t) \xi_{L}\left(t^{\prime}\right)^{T}\right\rangle=V^{C L}\left(\dot{g}\left(t, t_{0}\right)\left\langle u^{L}\left(t_{0}\right) u^{L}\left(t_{0}\right)^{T}\right\rangle \dot{g}\left(t^{\prime}, t_{0}\right)^{T}\right.
$$

$$
\begin{aligned}
& -\dot{g}\left(t, t_{0}\right)\left\langle u^{L}\left(t_{0}\right) \dot{u}^{L}\left(t_{0}\right)^{T}\right\rangle g\left(t^{\prime}, t_{0}\right)^{T} \\
& -g\left(t, t_{0}\right)\left\langle\dot{u}^{L}\left(t_{0}\right) u^{L}\left(t_{0}\right)^{T}\right\rangle \dot{g}\left(t^{\prime}, t_{0}\right)^{T} \\
& \left.+g\left(t, t_{0}\right)\left\langle\dot{u}^{L}\left(t_{0}\right) \dot{u}\left(t_{0}\right)^{T}\right\rangle g\left(t^{\prime}, t_{0}\right)^{T}\right) V^{L C} .(9)
\end{aligned}
$$

For a sensible heat bath, the correlation should be time translationally invariant and independent of $t_{0}$. Indeed, great simplification can be done if we use the eigenmode representation for the matrix $g$ :

$$
g\left(t, t^{\prime}\right)=S^{T} g^{d} S, \quad g_{j}^{d}=-\theta\left(t-t^{\prime}\right) \frac{\sin \omega_{j}\left(t-t^{\prime}\right)}{\omega_{j}},
$$

where $S$ is the orthogonal matrix that diagonalizes $K^{L}$, $S K^{L} S^{T}=\Omega^{2}, \Omega^{2}$ is a diagonal matrix with diagonal elements $\omega_{j}^{2} ; \omega_{j}$ s are the positive eigen frequencies. Substituting this result into the correlation expression, also using the quantum equilibrium correlation values for $\left\langle u u^{T}\right\rangle$, $\left\langle\dot{u} u^{T}\right\rangle,\left\langle u \dot{u}^{T}\right\rangle$, and $\left\langle\dot{u} \dot{u}^{T}\right\rangle$, we obtain

$$
\left\langle\xi_{L}(t) \xi_{L}\left(t^{\prime}\right)^{T}\right\rangle=V^{C L} S^{T} D S V^{L C},
$$

where $D$ is a diagonal matrix with elements $D_{j}=$ $\left(2 f\left(\omega_{j}\right)+1\right) \frac{\hbar}{2 \omega_{j}} \cos \omega_{j}\left(t-t^{\prime}\right)+\frac{\hbar}{2 i \omega_{j}} \sin \omega_{j}\left(t-t^{\prime}\right) . f(\omega)=$ $\left[\exp \left(\beta_{L} \hbar \omega\right)-1\right]^{-1}$ is the Bose-Einstein distribution function at the temperature of the left lead.

The appearance of the imaginary number in the last term in $D$ seems ominous, as we cannot simulate a heat bath with imaginary correlation. The imaginary part comes from the fact that in quantum mechanics, $\xi(t)$ and $\xi\left(t^{\prime}\right)$ are non-commuting, and the product of the two is not a Hermitian operator. Such a difficulty can be easily overcome if we use a symmetrized correlation $\frac{1}{2}\left\langle\xi_{j}(t) \xi_{l}\left(t^{\prime}\right)+\xi_{l}\left(t^{\prime}\right) \xi_{j}(t)\right\rangle$. This amounts to interchanging $t$ and $t^{\prime}$ and taking the transpose. The final effect is simply to drop the imaginary term.

Then the question arises that such a treatment will not give correctly the quantum results. It turns out that it causes no problem, at least for the expression of heat current. We can show rigorously that, with the symmetrized heat baths, we reproduce exactly the Landauer result with Caroli-formula as the transmission coefficient. However, the symmetrization does have a consequence to the quantum heat-current fluctuations.

Using the (surface) density of states, the expression can be further simplified to get a rather compact result for the spectrum of the noises [14],

$$
\tilde{F}[\omega]=\int_{-\infty}^{\infty}\left\langle\xi_{L}(t) \xi_{L}^{T}(0)\right\rangle e^{i \omega t} d t=\left(f_{L}(\omega)+\frac{1}{2}\right) \hbar \Gamma_{L}[\omega]
$$

where $\Gamma_{L}[\omega]=i\left(\Sigma_{L}[\omega]-\Sigma_{L}[\omega]^{\dagger}\right)=-2 \operatorname{Im} V^{C L} g[\omega] V^{L C}$. The spectrum function $\tilde{F}[\omega]$ is even in $\omega$ and is a symmetric matrix. Classical limit is obtained if we take $(f(\omega)+1 / 2) \hbar \approx k_{B} T_{L} / \omega$, where $k_{B}$ is the Boltzmann constant and $T_{L}$ is the temperature of left lead. 
The thermal current in steady state can be computed in several equivalent ways:

$$
\begin{aligned}
I_{L}=-I_{R}= & -\left\langle\frac{d H_{L}}{d t}\right\rangle=\left\langle\left(\dot{u}^{L}\right)^{T} V^{L C} u^{C}\right\rangle \\
& =-\left\langle u^{C}(t)^{T} \dot{B}(t)\right\rangle=\left\langle\dot{u}^{C}(t)^{T} B(t)\right\rangle,
\end{aligned}
$$

where $B(t)=-\int_{t_{0}}^{t} \Sigma_{L}\left(t, t^{\prime}\right) u^{C}\left(t^{\prime}\right) d t^{\prime}+\xi_{L}(t)$.

The stochastic differential equation, Eq. (7), can be solved numerically in a straightforward way. Both the memory function (retarded self-energy $\Sigma$ ) and noise spectrum $\tilde{F}$ can be obtained through the surface Green's function $g$. Efficient recursive algorithms exist for the solution of $g$ [10, 18]. A set of past coordinates, $u^{C}(t)$, needs to be stored, in order to perform a numerical integration due to the self-energy. We can use a simple rectangular rule for the integration. The random noises can be generated using a spectrum method [19]. Let the discrete Fourier transform of $\xi(t)$ be $\eta_{k}=\eta_{-k}^{*}=$ $a_{k}+i b_{k}, k=-M / 2, \cdots,-1,0,1, \ldots, M / 2-1$; where $M$ is the number of sampling points in the discrete Fourier transform. Then the noises can be generated by taking real numbers $a_{k}$ and $b_{k}(k>0)$ as independent Gaussian random numbers with zero mean and variance $\frac{1}{2} \tilde{F}\left[\omega_{k}\right] h M$, where $h$ is the integration step size, $\omega_{k}=2 \pi k /(h M)$. The noise values at required times are obtained by an inverse fast Fourier transform as $\xi(t=h l)=\frac{1}{h M} \sum_{k} \eta_{k} \exp (-i 2 \pi l k / M)$. The numerical integration of Eq. (7) is not substantially more expensive than standard MD. This is because the forces are usually short-ranged; we only need to do the extra work for these sites that are directly connected to the leads. Note that the matrix elements of $V^{C L}$ and $V^{C R}$ are zero except those that have a direct connection between the center and leads. The computational complexity becomes more favorable as the system becomes larger.

To illustrate the general method, we consider a simple 1D model with a quartic on-site potential ( $\phi^{4}$ model). Such a model is known to have diffusive transport in the classical limit [20]. The equation of motion is given by

$$
\ddot{u}_{j}=K u_{j-1}-\left(2 K+K_{0}\right) u_{j}+K u_{j+1}-\mu_{j} u_{j}^{3},
$$

where the nonlinear term is nonzero only in the central region, i.e., $\mu_{j}=\mu$ if $1 \leq j \leq N$ and $\mu_{j}=0$ otherwise. The required surface Green's function can be obtained analytically in frequency domain as $g[\omega]=-\lambda / K$, where $\lambda$ is the root of the quadratic equation, $K \lambda^{-1}+(\omega+$ $\left.i 0^{+}\right)^{2}-2 K-K_{0}+K \lambda=0$, such that $|\lambda|<1$. We use the following expression for the heat current [3],

$$
I^{\mathrm{MD}}=\frac{K}{2}\left\langle\left(\dot{u}_{j}+\dot{u}_{j+1}\right)\left(u_{j}-u_{j+1}\right)\right\rangle .
$$

Due to energy conservation along the chain, one can show that Eq. (15) is equal to that defined by Eq. (13) in steady state.

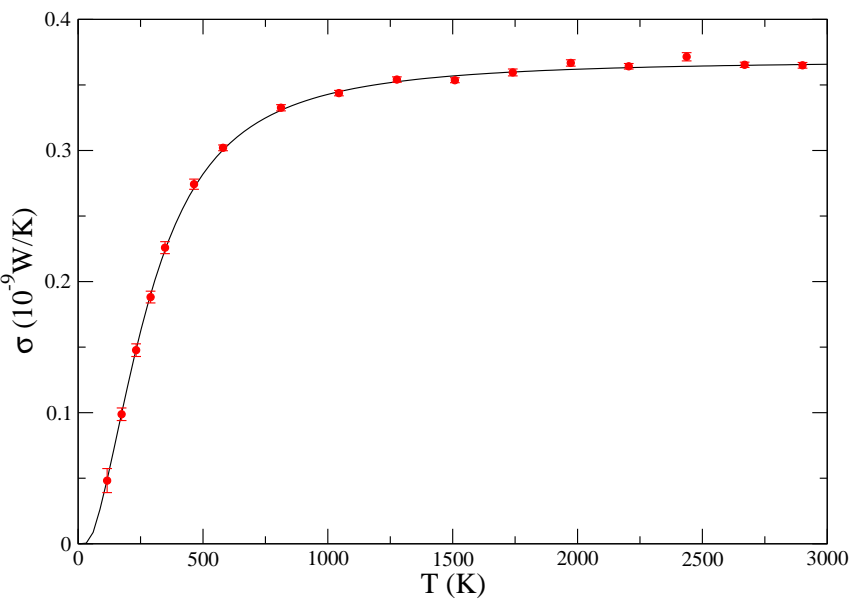

FIG. 1: Thermal conductance $\sigma$ for the 1D onsite model without the nonlinear interaction, with spring constant $K=$ $1.0 \mathrm{eV} /\left(\mathrm{amu} \AA^{2}\right), K_{0}=0.1 K$. The smooth curve is the Landauer formula result, while the symbols are MD results with a size $N=8$. The time-step $h=10^{-16}$ s and $5 \times 10^{8} \mathrm{MD}$ steps each are used.

We now present our numerical results. First, when there is no nonlinear interaction, $\mu_{j} \equiv 0$, the heat current can be computed exactly through the Landauer/Caroli formula, $I_{L}=\frac{1}{2 \pi} \int_{0}^{\infty} d \omega \hbar \omega \operatorname{Tr}\left(G^{r} \Gamma_{L} G^{a} \Gamma_{R}\right)\left(f_{L}-f_{R}\right)$, where $G^{r}=\left(G^{a}\right)^{\dagger}=\left(\left(\omega+i 0^{+}\right)^{2}-K^{C}-\Sigma\right)^{-1}$. The molecular dynamics with the quantum heat bath reproduces this result exactly. In Fig. 1, we present the comparison of MD and the exact curve. The conductance is defined by

$$
\sigma=\lim _{T_{L} \rightarrow T_{R}} \frac{I_{L}}{T_{L}-T_{R}} .
$$

A numerical finite-difference with 10-percent above or below the average temperature is used. Within MD statistical errors (computed from statistical fluctuations of multiple runs), the agreement is perfect. For the ballistic transport, the thermal conductance is independent of the lengths $N$ of the system.

A nontrivial result is obtained when the system has nonlinear interactions. This is presented in Fig. 2, With a nonlinear strength of $\mu=1\left[\mathrm{eV} /\left(\mathrm{amu}^{2} \AA^{4}\right)\right]$, we obtain quantitatively correct picture of ballistic transport at low temperatures and small sizes $\left(\sigma \propto N^{0}\right)$ and diffusive transport at high temperatures and large sizes $(\sigma \propto 1 / N)$. The low-temperature results can be compared with the NEGF ones. This is presented as smooth curves in Fig. 2, The NEGF results are obtained with a mean-field approximation to the self-energies [10]. The Green's functions are iterated in equilibrium and the conductance is calculated with an approximate formula for the transmission, $\tilde{T}[\omega]=\frac{1}{2} \operatorname{Tr}\left\{G^{r}\left(\Gamma_{L}+\frac{1}{2} \Gamma_{n}\right) G^{a} \Gamma_{R}\right\}+$ $\frac{1}{2} \operatorname{Tr}\left\{G^{a} \Gamma_{L} G^{r}\left(\Gamma_{R}+\frac{1}{2} \Gamma_{n}\right)\right\}$, where the nonlinear effect is reflected in the extra nonlinear self-energy, $\Gamma_{n}=$ 


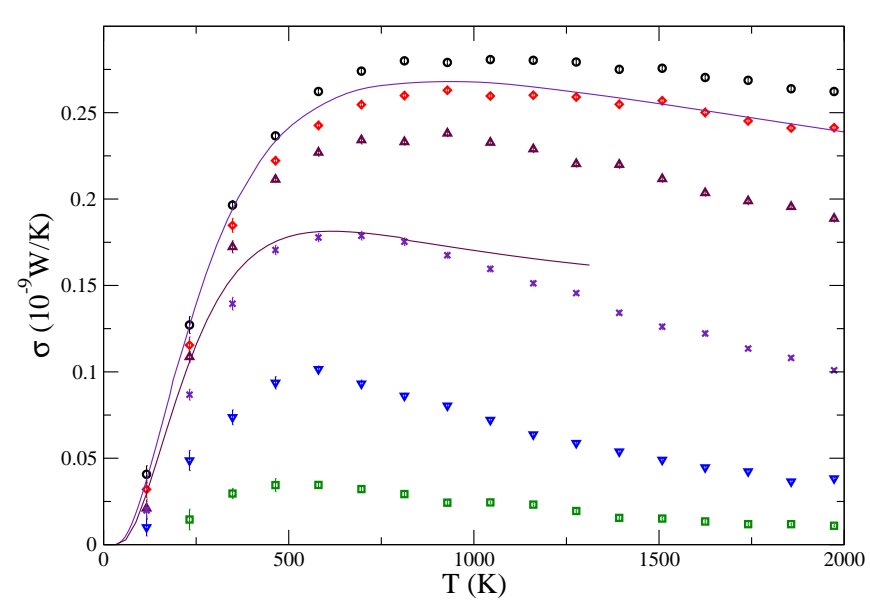

FIG. 2: Thermal conductance $\sigma$ for the 1D onsite model with the nonlinear interaction $\mu=1 \mathrm{eV} /\left(\mathrm{amu}^{2} \AA^{4}\right)$, spring constant $K=1.0 \mathrm{eV} /\left(\mathrm{amu} \AA^{2}\right), K_{0}=0.1 K$. The smooth curves are the NEGF results for sizes $N=4$ and 32, respectively, while the symbols are MD results with size $N$ from 4, 16, 64, 256, 1024, to 4096 , from top to bottom. The time-step $h=10^{-16} \mathrm{~s}$ and $10^{8}$ MD steps each are used.

$i\left(\Sigma_{n}^{r}-\Sigma_{n}^{a}\right)$. The MD and NEGF results agree with each other at the low-temperature side very well. Clearly, the nonlinear NEGF results are not exact at high temperatures. Thus the deviation between MD and NEGF is understandable. If classical heat baths are used, then as the temperature decreases, the thermal conductance increases monotonically to a size-independent ballistic value of $\left(\omega_{\max }-\omega_{\min }\right) k_{B} /(2 \pi)$, where $\omega_{\max }-\omega_{\min }$ is the phonon band width. At the intermediate range of temperatures, no reliable methods exist that can be compared with the quantum MD results. Thus, in this difficult temperature range, the MD results are the only numbers to offer. Whether we see ballistic or diffusive transport in a given temperature is determined by the mean free path of the phonons in comparison with the system size $N$. From the data in Fig. 2, we can judge that the mean free path is about $10^{3}$ lattice spacings in temperature range of $1000 \mathrm{~K}$.

The dynamics also gives correctly the quantum average energy and quantum heat capacity (say, with equal temperatures for the two leads). This is consistent with the fact that quantum conductance is calculated correctly. In classical simulation, the average kinetic energy gives the local temperature of the system, $\left\langle\dot{u}_{j}^{2}\right\rangle=k_{B} T$. However, this is not true in our dynamics and the kinetic energy is several times larger than implied by the equipartition theorem. Interestingly, in the limit of high temperatures of several thousand Kelvin, the equipartition theorem is restored.

In summary, we showed that a generalized Langevin dynamics as a classical stochastic differential equation can reproduce quantum ballistic transport if the heat baths follow the quantum prescription. This is achievable because there is very little difference between a quantum and classical system if the system is linear. The dynamics is such that it smoothly crosses over to the classical regime. Thus the method produces correctly results both in the quantum ballistic limit and classical diffusive limit. We have applied the method to a simple 1D onsite model. Clearly, it is of general applicability. For example, we can use the approach to study ballistic and diffusive thermal transport in carbon nanotubes and graphene ribbons. We can also study the nonlinear effect in interfaces. The present method opens new way for studying quantum transport and nonlinearity.

The author thanks Jingtao Lü, Jian Wang and Imam Makhfudz for discussions. The computations were performed on the clusters of the Center for Computational Science and Engineering and of Singapore-MIT Alliance, as well as on IBM cluster of Institute of High Performance Computing. This work is supported in part by a Faculty Research Grant of the National University of Singapore.

* Also affiliated with Singapore-MIT Alliance, 4 Engineering Drive 3, Singapore 117576; and Institute of High Performance Computing, 1 Science Park Road, Singapore 117528.

[1] R. E. Peierls, Quantum Theory of Solids, Chap. 2, (Oxford University Press, 1955).

[2] P. Carruthers, Rev. Mod. Phys. 33, 92 (1961).

[3] S. Lepri, R. Livi, and A. Politi, Phys. Rep. 377, 1 (2003).

[4] A. J. H. McGaughey and M. Kaviany, Adv. in Heat Transfer, 39, 169 (2006).

[5] J.-S. Wang and B. Li, Phys. Rev. E 70, 021204 (2004).

[6] A. Ozpineci and S. Ciraci, Phys. Rev. B 63, 125415 (2001).

[7] N. Mingo and L. Yang, Phys. Rev. B 68, 245406 (2003).

[8] T. Yamamoto and K. Watanabe, Phys. Rev. Lett, 96 255503 (2006).

[9] A. Dhar and D. Sen, Phys. Rev. B 73, 085119 (2006).

[10] J.-S. Wang, J. Wang, and N. Zeng, Phys. Rev. B 74, 033408 (2006); J.-S. Wang, N. Zeng, J. Wang, C.-K. Gan, Phys. Rev. E, accepted,(2007).

[11] N. Mingo, Phys. Rev. B 74, 125402 (2006).

[12] M. Michel, G. Mahler, and J. Gemmer, Phys. Rev. Lett. 95, 180602 (2005).

[13] D. Segal, A. Nitzan, and P. Hänggi, J. Chem. Phys. 119, 6840 (2003).

[14] A. Dhar and D. Roy, J. Stat. Phys. 125, 801 (2006).

[15] H. Spohn, J. Stat. Phys, 124, 1041 (2006); K. Aoki, J. Lukkarinen, and H. Spohn, J. Stat. Phys. 124, 1105 (2006).

[16] K. Lindenberg and B. J. West, The Nonequilibrium Statistical Mechanics of Open and Closed Systems (VCH Publishers, New York, 1990), p.194.

[17] P. Hänggi and G.-L. Ingold, Chaos, 15, 026105 (2005).

[18] M. P. López Sancho, J. M. López Sancho, and J. Rubio, J. Phys. F: Met. Phys. 15, 851 (1985).

[19] J. García-Ojalvo and J. M. Sancho, Noise in Spatially Extended Systems (Springer-Verlag, New York, 1999). 
[20] B. Hu, B. Li, and H. Zhao, Phys. Rev. E 61, 3828 (2000). 